\title{
Microstructure and Dynamics of Wormlike Micellar Solutions Formed by Mixing Cationic and Anionic Surfactants
}

\author{
Richard D. Koehler \\ DuPont Central Research and Development, Experimental Station, P.O. Box 80328, \\ Wilmington, Delaware 19880-0328 \\ Srinivasa R. Raghavan and Eric W. Kaler* \\ Center for Molecular and Engineering Thermodynamics, Department of Chemical Engineering, \\ University of Delaware, Newark, Delaware 19716
}

Received: May 23, 2000; In Final Form: September 19, 2000

\begin{abstract}
Small-angle neutron scattering (SANS) and rheology are used to probe the wormlike micelles formed in mixtures of a cationic (cetyl trimethylammonium tosylate, CTAT) and an anionic surfactant (sodium dodecyl benzene sulfonate, SDBS). For a fixed composition of 97/3 CTAT/SDBS, the zero-shear viscosity $\eta_{0}$ initially increases rapidly with surfactant concentration, but decreases beyond an intermediate concentration $\phi_{\max }$. The solutions show a scattering peak in SANS and the height of the scattering peak also exhibits a maximum around $\phi_{\max }$. These results are interpreted in terms of a maximum in the linear micellar contour length $\bar{L}$ at $\phi_{\max }$, and suggest that the hydrodynamic and electrostatic correlation lengths reach an optimal ratio at this point. For a fixed total surfactant concentration, the viscosity $\eta_{0}$ also reaches a maximum at an intermediate SDBS fraction. The decrease in $\eta_{0}$ at high SDBS fractions is interpreted in terms of the polyelectrolyte nature of the micelles and the increased chain flexibility caused by the rising ionic strength of the solutions. An alternate possibility may involve a progression from linear to branched micelles with increasing SDBS content.
\end{abstract}

\section{Introduction}

Ionic surfactants in aqueous solution can self-assemble into long flexible wormlike or threadlike micelles. ${ }^{1-3}$ These micelles are generally formed in the presence of high concentrations of salt which screens the electrostatic repulsions between the charged surfactant headgroups. ${ }^{2,3}$ Typical examples include the cationic surfactant, cetyl trimethylammonium bromide (CTAB), and analogues of CTAB with hydrophobic counterions such as salicylate, tosylate, or dichlorobenzoate. In the latter cases, wormlike micelles are formed even in the absence of salt, with the counterions apparently inserting between the headgroups and facilitating micellar growth. ${ }^{4-8}$

This study focuses on one such cationic surfactant, cetyl trimethylammonium tosylate (CTAT), and we report here the effects of incorporating small amounts of an anionic surfactant, sodium dodecyl benzene sulfonate (SDBS), into salt-free solutions of CTAT. The addition of oppositely charged SDBS to CTAT is expected to enhance the formation of wormlike micelles because SDBS reduces the micellar surface potential via charge neutralization and also increases the ionic strength by virtue of the released counterions. ${ }^{9,10}$ Thus, a mixture of oppositely charged surfactants can show a synergistic enhancement of rheological properties over that of either parent surfactant, an effect which may be exploited in applications. ${ }^{10,11}$ Few rheological studies, however, have focused on such mixed surfactant systems.

The phase diagram of the CTAT/SDBS system (Figure 1) is known. ${ }^{9}$ Rodlike or wormlike micelles are formed only in the

* Author to whom correspondence should be addressed. Tel.: (302) 8313553. Fax: (302) 831-1048. E-mail: kaler@che.udel.edu.

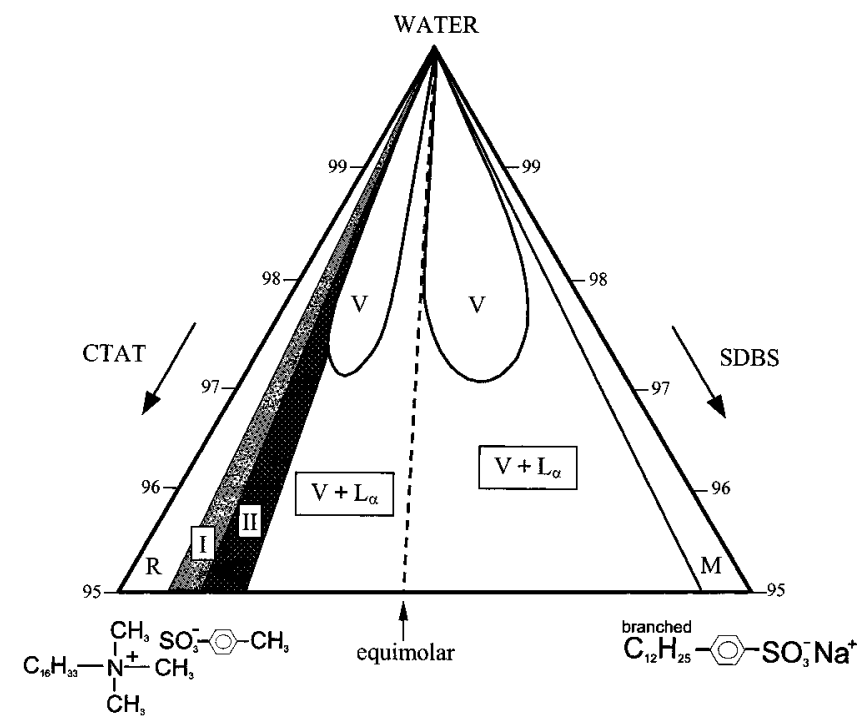

Figure 1. Water-rich corner of the phase diagram of CTAT/SDBS in $\mathrm{H}_{2} \mathrm{O}$. A rodlike micellar phase $(\mathrm{R})$ is present on the CTAT-rich side, and adjacent to this are two micelle/vesicle two-phase coexistence regions (I, II). There are also two vesicle lobes symmetrical around the equimolar line. Spherical micelles (M) are found in the SDBS-rich corner of the diagram.

CTAT-rich corner of the phase diagram (marked R)-at higher SDBS concentrations, spontaneous vesicles (V), and lamellar structures $\left(\mathrm{L}_{\alpha}\right)$ occur, and in the SDBS corner, spherical micelles are found. ${ }^{9}$ In $\mathrm{D}_{2} \mathrm{O}$ the vesicle regions are much smaller and the boundary of the CTAT-rich micellar phase is slightly shifted. ${ }^{9,10}$ The emphasis of this paper is the characterization of the mixed cationic/anionic wormlike micelles in the CTAT- 
rich micellar phase through a combination of rheology and small-angle neutron scattering (SANS). We focus on the semidilute regime of the solutions (above the overlap concentration $\left.c^{*}\right)$ in which the wormlike micelles are entangled into a mesh-like network.

Theory. A mean-field thermodynamic treatment of wormlike micelles predicts an exponential distribution of micellar lengths, with the average contour length $\bar{L}$ varying with surfactant volume fraction $\phi$ as: ${ }^{1}$

$$
\bar{L} \sim \phi^{1 / 2} \exp \left[E_{\mathrm{c}} / 2 k_{\mathrm{B}} T\right]
$$

Here, $E_{\mathrm{c}}$ is the energy required to create two end caps, and $k_{\mathrm{B}} T$ is the thermal energy. The above relation is only valid for electrostatically screened micelles. As micelles grow, they begin to entangle, and in the semidilute regime their rheology can be described by the Cates model. ${ }^{1,12-15}$ In this model, the reversible nature of the micelles allows scission (breaking) and recombination processes to act in addition to micellar reptation. The behavior of the micellar solutions is determined by the ratio of the characteristic breaking time $\left(\tau_{\mathrm{b}}\right)$ to the characteristic time taken by a micelle of mean length $\bar{L}$ to reptate $\left(\tau_{\text {rep }}\right) .{ }^{1}$ When breaking occurs often over the time scale of reptation $\left(\tau_{\text {rep }} \gg\right.$ $\tau_{\mathrm{b}}$ ), the solution behaves as a Maxwell fluid with a single relaxation time $\left(t_{\mathrm{R}}\right)$ given by ${ }^{1}$

$$
t_{\mathrm{R}}=\left(\tau_{\mathrm{b}} \tau_{\text {rep }}\right)^{1 / 2}
$$

Under oscillatory shear, the elastic modulus $G^{\prime}$ and the viscous modulus $G^{\prime \prime}$ for a Maxwell fluid obey the following relations as a function of the oscillation frequency $\omega::^{15}$

$$
G^{\prime}(\omega)=\frac{G_{\infty}^{\prime} \omega^{2} t_{\mathrm{R}}^{2}}{1+\omega^{2} t_{\mathrm{R}}^{2}} \quad G^{\prime \prime}(\omega)=\frac{G_{\infty}^{\prime} \omega t_{\mathrm{R}}}{1+\omega^{2} t_{\mathrm{R}}^{2}}
$$

Here, $G_{\infty}^{\prime}$ is the high-frequency plateau modulus, and the relaxation time $t_{\mathrm{R}}$ can be estimated as $\left(\omega_{\mathrm{c}}\right)^{-1}$ where $\omega_{\mathrm{c}}$ is the frequency where the two moduli crossover. Equivalently, a Cole-Cole plot $\left(G^{\prime \prime}\right.$ vs $\left.G^{\prime}\right)$ for a Maxwell fluid describes a semicircle. ${ }^{1,15}$

The rheology of wormlike micelles deviates from Maxwellian behavior at high frequencies, showing an upturn in the viscous modulus $G^{\prime \prime}$. These deviations result from a transition in the relaxation mode from reptation at longer time-scales to "breathing" or Rouse modes at short time-scales. The frequency at which $G^{\prime \prime}$ shows a deviation is $\approx 1 / \tau_{\mathrm{b}}$, where $\tau_{\mathrm{b}}$ is the micellar breaking time. ${ }^{1}$ The value of $G^{\prime \prime}$ at the minimum, i.e., $G_{\mathrm{min}}^{\prime \prime}$, can be related to the micellar contour length $\bar{L}$ via ${ }^{13}$

$$
\frac{G_{\min }^{\prime \prime}}{G_{\infty}^{\prime}} \approx \frac{l_{\mathrm{e}}}{\bar{L}}
$$

This equation allows the micellar contour length $\bar{L}$-an important and otherwise inaccessible quantity-to be estimated from rheological measurements. To do this, the entanglement length $l_{\mathrm{e}}$, i.e., the average contour length between two entanglement points, must first be determined according to $\mathrm{o}^{4,16}$

$$
l_{\mathrm{e}} \approx \frac{\xi_{\mathrm{H}}^{5 / 3}}{l_{\mathrm{p}}^{2 / 3}}
$$

Here, $l_{\mathrm{p}}$ is the persistence length of the micelles. The micelles act as rigid entities over length scales $\sim l_{\mathrm{p}}$ and are flexible at longer length scales (typically $\bar{L} \gg l_{\mathrm{p}}$ ). The parameter $\xi_{\mathrm{H}}$ is a hydrodynamic correlation length, typically identified with the network mesh size. It can be estimated from the plateau modulus $G_{\infty}^{\prime} \operatorname{via}^{16}$

$$
G_{\infty}^{\prime} \approx \frac{k_{\mathrm{B}} T}{\xi_{\mathrm{H}}^{3}}
$$

Scaling relations can be deduced for key rheological quantities as a function of surfactant volume fraction $\phi$. For the plateau modulus $G_{\infty}^{\prime}$ and the reptation time $\tau_{\text {rep}}$, the results are the same as for polymer solutions: ${ }^{16}$

$$
\begin{gathered}
G_{\infty}^{\prime} \sim \phi^{9 / 4} \\
\tau_{\text {rep }} \sim \bar{L}^{3} \phi^{3 / 2}
\end{gathered}
$$

The breaking time of a micelle is assumed to be independent of surfactant concentration and varies inversely with micellar length, ${ }^{12}$ i.e., $\tau_{\mathrm{b}} \sim(\bar{L})^{-1}$. This assumption, when combined with eqs 8,1 , and 2 , yields the relaxation time $t_{\mathrm{R}}$ in the fast-breaking limit. In turn, the zero-shear viscosity $\eta_{0}$ of a Maxwell fluid, which is the product of $t_{\mathrm{R}}$ and $G_{\infty}^{\prime}$, can be obtained. The resultant scaling relations are ${ }^{1}$

$$
\begin{gathered}
t_{\mathrm{R}} \sim \bar{L} \phi^{3 / 4} \sim \phi^{1.25} \\
\eta_{0}=G_{\infty}^{\prime} t_{\mathrm{R}} \sim \bar{L} \phi^{3} \sim \phi^{3.5}
\end{gathered}
$$

Rheology of Wormlike Micelles. Experiments on some model systems, such as CTAB micelles in $0.25 \mathrm{M} \mathrm{KBr}$ have verified many of the above theoretical predictions, including the exponent of 3.5 for $\eta_{0} \cdot{ }^{17}$ However, significant discrepancies from this "classical picture" have also emerged, as briefly discussed below. Further details can be found elsewhere. ${ }^{2-4,18-20}$

The first set of anomalous findings pertain to the effect of salt concentration $c_{\mathrm{s}}$ on the rheology. Several cationic surfactant solutions show maxima in viscosity $\left(\eta_{0}\right)$ as a function of $c_{\mathrm{S}}$ at a constant surfactant concentration $c_{\mathrm{D}} \cdot{ }^{18,21-24}$ The solutions behave as Maxwell fluids beyond the viscosity maximum, and the plateau modulus $G_{\infty}^{\prime}$ is independent of $c_{\mathrm{s}}$. These effects are typically explained using the hypothesis of micellar branching at high salt. The "cross-links" in the resulting multi-connected micellar network can slide along the micelles and hence serve as stress release points. Such a micellar network will therefore show a reduced viscosity compared to entangled linear micelles. ${ }^{25}$ Thus, the maximum in $\eta_{0}$ with increasing $c_{\mathrm{s}}$ is supposed to signify a shift from linear to branched micelles. Branching is expected to occur when the free energy cost associated with cross-link formation becomes comparable to that for end caps. ${ }^{26}$ Some evidence for branched micelles has been provided by cryoTEM microscopy. ${ }^{27-29}$

The second class of anomalous findings deals with the effect of surfactant concentration $c_{\mathrm{D}}$ on the rheology. Cationic surfactants that can form wormlike micelles in the absence of salt (e.g., cetyl trimethylammonium salicylate) typically show a maximum in rheological properties $\left(t_{\mathrm{R}}, \eta_{0}\right)$ with increasing $c_{\mathrm{D}} \cdot{ }^{3-8}$ Viscosity maxima as a function of $c_{\mathrm{D}}$ are also found at constant $c_{\mathrm{S}} / c_{\mathrm{D}}$ for certain surfactant/salt pairs (e.g., cetyl trimethylammonium chloride/sodium tosylate). ${ }^{3,4,24}$ In all these cases, the plateau modulus $G_{\infty}^{\prime}$ steadily rises with surfactant concentration over the entire range and conforms to eq 6 . At the same time, the parameter $G_{\mathrm{min}}^{\prime \prime} / G_{\infty}^{\prime}$ describes a minimum as a function of $c_{\mathrm{D}}$. These findings have been interpreted in terms of a 
maximum in the micellar length $\bar{L}^{4,5}$ or alternately, in terms of micellar branching beyond the $\eta_{0}$ maximum. ${ }^{8}$ Neither phenomenon is understood quantitatively.

A limited number of rheological studies have been performed on mixed surfactant systems containing wormlike micelles. ${ }^{30-32}$ Herb et $a{ }^{30}$ reported interesting results for mixtures of a nonionic and an anionic surfactant. At a constant total surfactant concentration, the viscosity $\left(\eta_{0}\right)$ showed a maximum as the mole fraction of anionic surfactant was varied. For a constant ratio of the surfactants, the viscosity again described a maximum as a function of the total surfactant concentration. These results are analogous to the effects observed for single surfactantsthe first case corresponding to the salt effect and the second to the concentration effect. Hoffmann et al. $^{31}$ reported similar findings for mixtures of a zwitterionic surfactant with either a cationic or anionic surfactant. The data in the above studies were not analyzed using the Cates framework.

Synergism in cationic/anionic surfactant mixtures is wellknown. ${ }^{10,33}$ At low concentrations, micellar aggregation numbers increase as surfactant of opposite charge is added, and this is reflected in higher viscosities. ${ }^{34,35}$ Few systematic studies at higher concentrations have been conducted thus far, possibly because these mixtures often exhibit phase separation or precipitation even at low concentrations. This study is the first detailed exploration of the high concentration $\left(c>c^{*}\right)$ regime for cationic/anionic wormlike micelles using both rheology and SANS.

\section{Experimental Section}

Materials. Cetyl trimethylammonium tosylate (CTAT) was obtained from Sigma and was recrystallized from a 50/50 ethanol/acetone mixture. Soft-type (linear chain) sodium dodecyl benzene sulfonate (SDBS) was used as received from TCI. Surfactant solutions were prepared in distilled-deionized water. For the SANS experiments, samples were prepared in $\mathrm{D}_{2} \mathrm{O}$ (deuteration $99.9 \%$, low paramagnetism, low conductivity) purchased from Cambridge Isotope Laboratory.

Small-Angle Neutron Scattering (SANS). SANS experiments were performed on the NG-7 and NG-3 spectrometers at the Cold Neutron Research Facility of the National Institute of Standards and Technology (NIST) in Gaithersburg, MD. The incident neutron wavelength was $\lambda=6 \AA$ with a spread in wavelength $\Delta \lambda / \lambda$ of $15 \%$. Quartz cells with $2 \mathrm{~mm}$ path lengths were used and the samples were placed in a temperaturecontrolled chamber held at $25 \pm 0.1{ }^{\circ} \mathrm{C}$. The scattering spectra were corrected for background radiation, detector efficiency, empty cell scattering, and sample transmission. The spectra were radially averaged and placed on an absolute scale using calibration standards provided by NIST.

Rheology. Steady and dynamic rheological experiments were performed on a Rheometrics SR 500 stress-controlled rheometer. A couette geometry with a $32 \mathrm{~mm}$ diameter cup and a bob of $29.5 \mathrm{~mm}$ diameter and $44.5 \mathrm{~mm}$ length was used. The frequency spectra were conducted in the linear viscoelastic regime of the samples, as determined previously by dynamic stress sweep measurements. For the steady-shear experiments, an equilibration time of $90 \mathrm{~s}$ was given at each data point; creep experiments indicated this amount of time to be sufficient for attainment of equilibrium at the shear stresses investigated. All experiments were performed at $25 \pm 0.1{ }^{\circ} \mathrm{C}$

\section{Results}

The CTAT-rich wormlike micellar region extends from the pure CTAT/water axis to approximately 90/10 CTAT/SDBS,
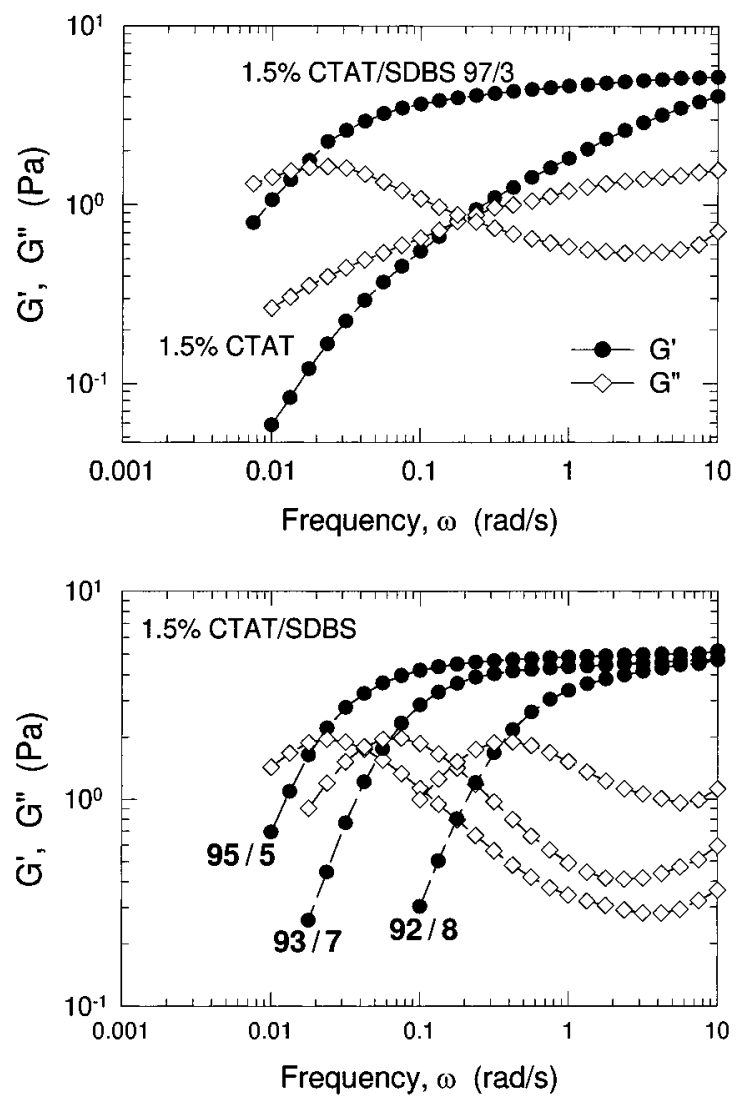

Figure 2. Effect of varying the ratio of CTAT to SDBS on the dynamic frequency response. The total surfactant concentration is held constant at $1.5 \mathrm{wt} \%$.

at which point there is a transition to a two-phase rodlike micelle/vesicle region (Figure 1). ${ }^{9,10}$ The wormlike micellar solutions are clear and their viscoelasticity is evidenced from the recoil of trapped air bubbles in the sample. Such recoil is observed in samples containing as low as $0.25 \mathrm{wt} \%$ surfactant. The solutions also show streaming birefringence when viewed between crossed polarizers. ${ }^{11}$

3.1. Rheology. Effect of Surfactant Composition. The effect of progressively adding SDBS to CTAT was studied by varying the CTAT/SDBS ratio at a constant total surfactant concentration of $1.5 \mathrm{wt} \%$. Figure $2 \mathrm{a}$ compares the dynamic frequency response for pure CTAT with that for 97/3 CTAT/SDBS. The response for pure CTAT indicates a spectrum of relaxation times, with the longest relaxation time $t_{\mathrm{R}}$ being about $6 \mathrm{~s}$. On inclusion of just 3 parts SDBS, the relaxation spectrum narrows and shifts to longer time scales. Further increase in SDBS content leads to fluids with a single relaxation time and a monoexponential stress decay, i.e., Maxwell fluids (Figure 2b). Correspondingly, the zero-shear viscosity $\eta_{0}$ increases by an order of magnitude over that of pure CTAT (Figure 3a).

For SDBS fractions beyond an intermediate ratio of 96/4 CTAT/SDBS, however, $t_{\mathrm{R}}$ and $\eta_{0}$ begin to decrease (Figures $2 \mathrm{~b}$ and $3 \mathrm{~b}$ ). There is a drop in $\eta_{0}$ by nearly 3 orders of magnitude on proceeding from 96/4 to 91/9 CTAT/SDBS (Figure 4a). Increasing the SDBS fraction beyond 91/9 causes the samples to phase separate. In the regime of decreasing relaxation times, the solutions are Maxwell fluids. The plateau modulus $G_{\infty}^{\prime}$ remains virtually unchanged over the entire composition range while the parameter $G_{\text {min }}^{\prime \prime} / G_{\infty}^{\prime}$ shows a minimum with increasing SDBS content (Figure $4 \mathrm{~b}$ ). The main result here is the maxima in key rheological parameters $\left(t_{\mathrm{R}}, \eta_{0}\right)$ at an intermediate surfactant composition. 

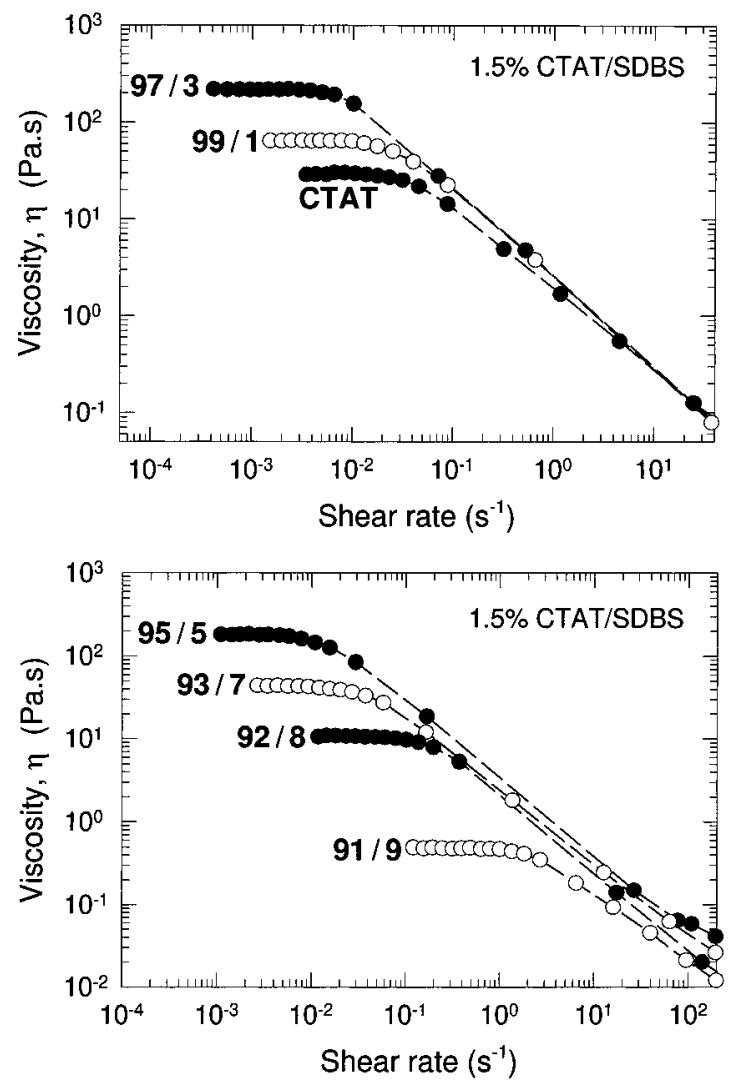

Figure 3. Effect of varying the ratio of CTAT to SDBS on the steadyshear response. The total surfactant concentration is held constant at $1.5 \mathrm{wt} \%$.

Effect of Surfactant Concentration. The effect of surfactant concentration $(0.25$ to $4.0 \mathrm{wt} \%)$ was studied at a fixed ratio of $97 / 3$ CTAT/SDBS. At low concentrations ( $0.75 \%$ or less), the samples show predominantly viscous behavior in dynamic shear $\left(G^{\prime \prime}>G^{\prime}\right.$ over the range of frequencies) (Figure $5 \mathrm{a}$ ). The $0.5 \%$ sample shows shear-thickening in steady shear (Figure 6a). Wormlike micellar solutions are known to shear-thicken at concentrations close to $c^{*}$, and the phenomenon has been extensively studied for pure CTAT solutions. ${ }^{36,37}$ The shearthickening disappears at $0.75 \%$ surfactant and the viscosity exhibits a Newtonian plateau followed by shear-thinning at higher shear rates (Figure 6a). Further addition of surfactant results in a dramatic enhancement of the zero-shear viscosity $\eta_{0}$. Concomitantly, the samples exhibit viscoelastic behavior in dynamic shear and the longest relaxation time $t_{\mathrm{R}}$ increases with concentration. The relaxation spectrum also begins to narrow and for concentrations higher than ca. $2 \%$ surfactant, the samples are Maxwell fluids with a single relaxation time. Figure $5 \mathrm{~b}$ illustrates the Maxwellian response of fluids with $2.5 \%$ and $4 \%$ surfactant.

Beyond an intermediate surfactant concentration (ca. 2\%), $t_{\mathrm{R}}$ and $\eta_{0}$ begin to decrease (Figures $5 \mathrm{~b}, 6 \mathrm{~b}$ ). A plot of these quantities against surfactant volume fraction $\phi$ (Figure 7a) shows a steep rise in $\eta_{0}$ at low $\phi$ according to a power law $\left(\eta_{0} \sim\right.$ $\phi^{9.1}$ ), while at high $\phi$ there is a drop in $\eta_{0}$ by a factor of about 3 . For charged micelles, the concentration at which a steep viscosity rise is initiated is the overlap concentration $\phi^{*}$ (the onset of the semidilute regime) and the relative viscosity $\eta_{0} / \eta_{\mathrm{s}}$ (where $\eta_{\mathrm{s}}$ is the solvent viscosity) is ca. 2 at this point. ${ }^{4,38}$ As the micelles grow longer, they eventually begin to entangle, and at the entanglement concentration $\phi_{\mathrm{e}}$ the relative viscosity $\eta_{0} / \eta_{\mathrm{s}}$ is ca. $50 .{ }^{15}$ From these considerations, $\phi^{*}$ and $\phi_{\mathrm{e}}$ are about 0.0027 and 0.0064 , respectively, for this system. The plateau
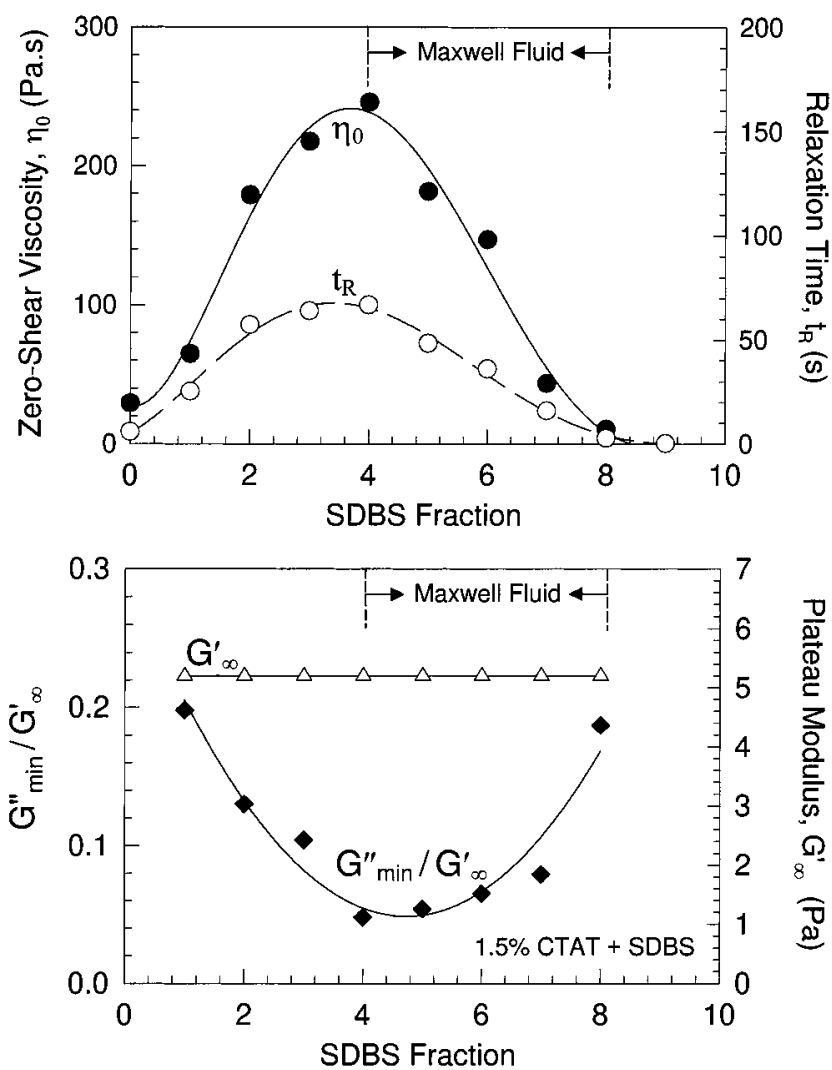

Figure 4. Rheological quantities as a function of the SDBS weight fraction in the added surfactant, i.e., $[\mathrm{SDBS}] /([\mathrm{CTAT}]+[\mathrm{SDBS}])$. The total surfactant concentration is constant at $1.5 \mathrm{wt} \%$.

modulus $G_{\infty}^{\prime}$ monotonically increases over the range of surfactant concentrations according to a power law: $G_{\infty}^{\prime} \sim \phi^{2.1}$ (Figure $7 \mathrm{~b}$ ). This is close to the theoretical value of 2.25 (eq 7). The ratio $G_{\mathrm{min}}^{\prime \prime} / G_{\infty}^{\prime}$, on the other hand, exhibits a minimum at an intermediate concentration (Figure 7b).

3.2. Small-Angle Neutron Scattering (SANS). Static SANS spectra for CTAT/SDBS solutions in $\mathrm{D}_{2} \mathrm{O}$ are shown in Figures 8 and 9 . The spectra are $\log -\log$ plots of the scattered intensity $I\left(\mathrm{~cm}^{-1}\right)$ on an absolute scale as a function of the scattering vector $q$, where

$$
q=\frac{4 \pi}{\lambda} \sin \left[\frac{\theta}{2}\right]
$$

Here $\lambda$ is the wavelength of the neutrons, and $\theta$ is the scattering angle. Qualitatively, the SANS spectra are typical of a wormlike micellar system with unscreened electrostatic interactions. ${ }^{39-43}$ The slope of -1 in the $\log -\log$ plot at intermediate $q$ (i.e., $I \sim$ $\left.q^{-1}\right)$ is a distinctive characteristic of long, flexible cylindrical micelles. The extent of this $q^{-1}$ region is quite small due to the existence of unscreened electrostatic interactions between the charged micelles. As a result of these interactions, the curves exhibit a scattering peak at low $q$. The data at high $q$ is consistent with scattering from the circular cross-section of the micelles. Shear-SANS measurements of these micellar solutions also support the presence of wormlike micelles that align in a flow. ${ }^{11}$

The effects of varying the surfactant composition from pure CTAT to $94 / 6$ CTAT/SDBS for a fixed total surfactant concentration $(1.5 \mathrm{wt} \%$ ) are shown in Figure 8. (Samples at SDBS content higher than $94 / 6$ were not studied since they form two phases in $\mathrm{D}_{2} \mathrm{O}$.) The scattering peak for CTAT is sharper and more intense than for the SDBS-containing samples, and as more SDBS is incorporated into the micelles the scattering 

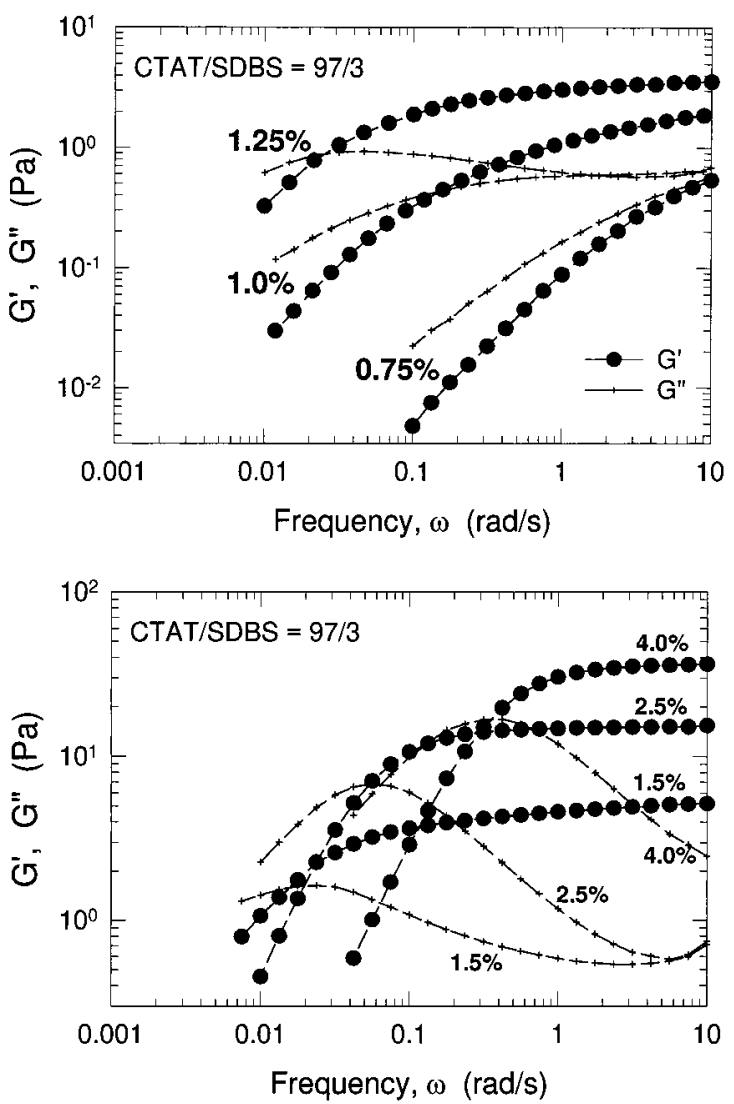

Figure 5. Effect of varying the total surfactant concentration on the dynamic frequency response. The weight ratio of CTAT to SDBS is held constant at $97 / 3$.

peak progressively becomes weaker. However, the peak position $\left(q_{\max }\right)$ does not change with surfactant composition (Figure 10). The height of the scattering peak can be characterized by the ratio $I_{\max } / I_{0}$, where $I_{\max }$ is the intensity at $q_{\max }$ and $I_{0}$ is the intensity in the plateau at $q \rightarrow 0$. The peak height drops exponentially with increasing SDBS content (Figure 10).

The effects of varying the surfactant concentration in the range $0.1-5$ wt $\%$ for a fixed ratio of 97/3 CTAT/SDBS are illustrated in Figure 9. At very low concentrations (0.1 wt \%) the data shows a monotonic decrease in $I$ vs $q$. A weak scattering peak first becomes noticeable at a concentration of $0.25 \mathrm{wt} \%$. As the concentration is increased further, the scattering peak becomes stronger and its position $\left(q_{\max }\right)$ shifts to higher $q$. The peak position varies with surfactant volume fraction $\phi$ as $q_{\max }$ $\sim \phi^{0.5}$ (Figure 11). The scattering peak height $\left(I_{\max } / I_{0}\right)$ shows a maximum as a function of surfactant volume fraction $\phi$ (Figure 11). The maximum occurs at approximately the same concentration at which the rheological properties are maximized.

The cross-sectional radius $r_{\mathrm{cs}}$ of the wormlike micelles can be obtained by analyzing the high- $q$ portions of the scattering plots using the Guinier approximation for the form factor: ${ }^{39}$

$$
q \cdot I(q) \sim \exp \left(-q^{2} R_{\mathrm{g}, \mathrm{cs}}^{2} / 2\right)
$$

Here, $R_{\mathrm{g}, \mathrm{cs}}$ is the cross-sectional radius of gyration of the cylindrical micelles. For a circular cross-section, the micellar radius $r_{\mathrm{cs}}$ is obtained as

$$
r_{\mathrm{cs}}=\sqrt{2} R_{\mathrm{g}, \mathrm{cs}}
$$

Cross-sectional Guinier plots $\left(\ln (I q)\right.$ vs $\left.q^{2}\right)$ are shown in Figure 12 for $1.5 \%$ CTAT and $1.5 \%$ 99/1 CTAT/SDBS in $\mathrm{D}_{2} \mathrm{O}$. From
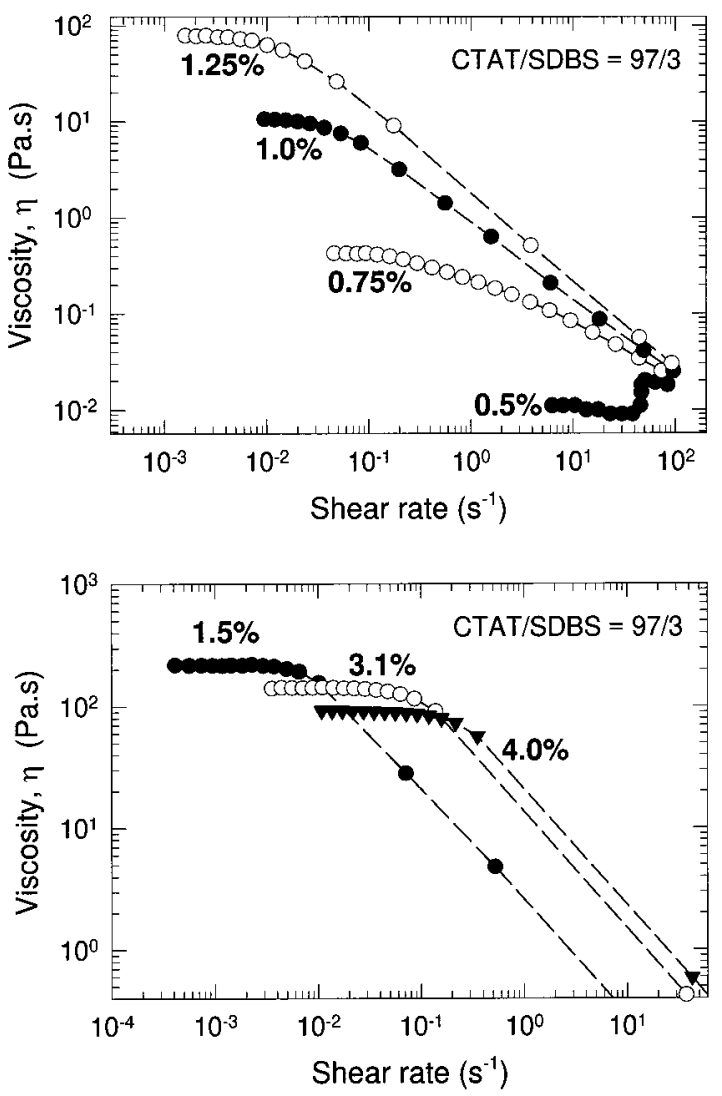

Figure 6. Effect of varying the total surfactant concentration on the steady shear response. The weight ratio of CTAT to SDBS is held constant at 97/3.

the fitted linear portions of the curves, the micellar radius $r_{\mathrm{cs}}$ is $22.5 \pm 0.5 \AA$, and this is the same for both micelles of pure CTAT and for the mixed CTAT/SDBS micelles. This value of $r_{\mathrm{cs}}$ is slightly less than the length of a fully extended CTAT molecule, which is ca. $25 \AA .44$ Thus, incorporation of low amounts of SDBS apparently does not alter the micellar radius.

\section{Discussion}

Our goal is to elucidate how the microstructure and rheology of these mixed micellar solutions evolve in response to changes in system constitution. The principal findings from rheology are the maxima in viscosity $\left(\eta_{0}\right)$ and relaxation time $\left(t_{\mathrm{R}}\right)$ as a function of both surfactant concentration and composition. Analogies with earlier findings are highlighted by organizing these results into two classes (Table 1). Class A refers to systems for which there is a viscosity maximum as a function of a compositional variable at constant surfactant concentration. These include the maxima as a function of salt in cationic surfactant systems and as a function of SDBS fraction in this study. In this case, the plateau modulus $G_{\infty}^{\prime}$ and the SANS peak position $q_{\max }$ remain constant even as the viscosity changes. Class B refers to systems for which there is a viscosity maximum as a function of surfactant concentration. In this case, there is a steady increase in the plateau modulus $G_{\infty}^{\prime}$ and the SANS peak position $q_{\max }$ accompanying the viscosity changes. The question then is whether there are common microstructural threads underlying these analogous effects.

Length Scales. The data admits characteristic microstructural length scales. First, the scattering peak position $q_{\max }$ dictates the correlation length $\xi=2 \pi / q_{\max }$ which represents the mean 

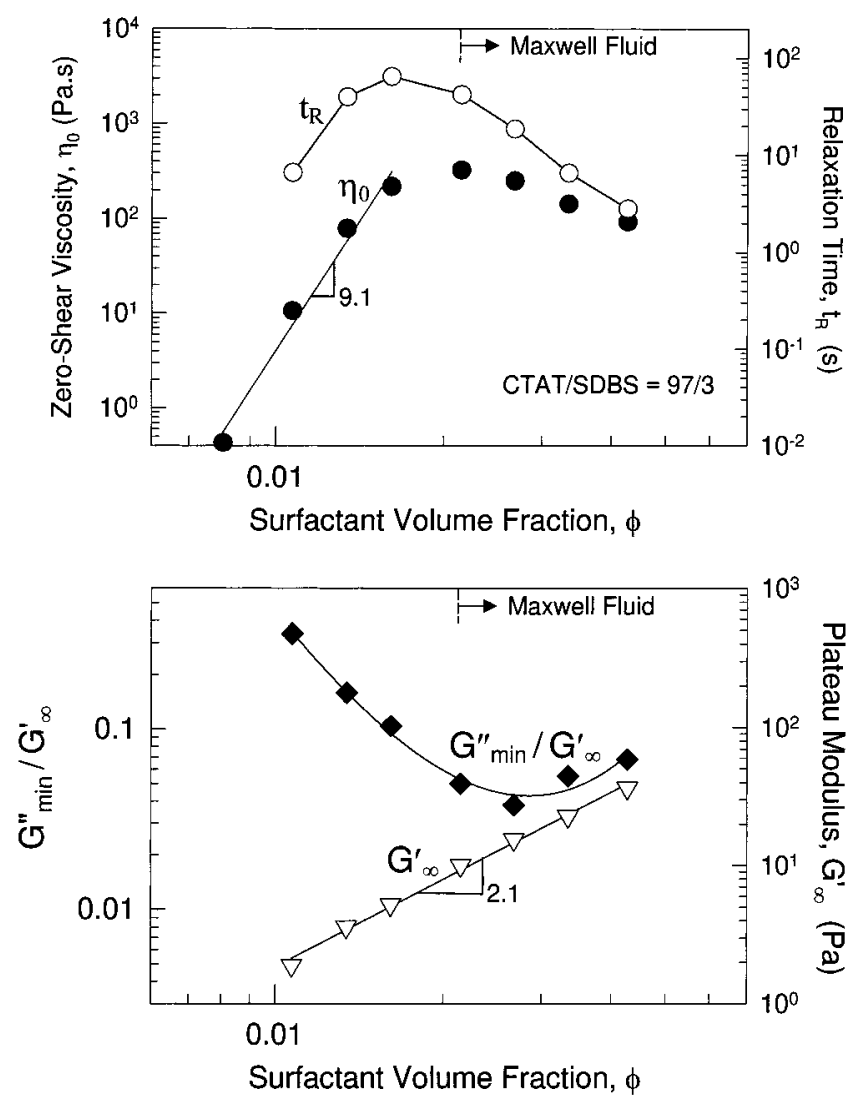

Figure 7. Rheological quantities as a function of surfactant concentration for a constant weight ratio of 97/3 CTAT/SDBS.

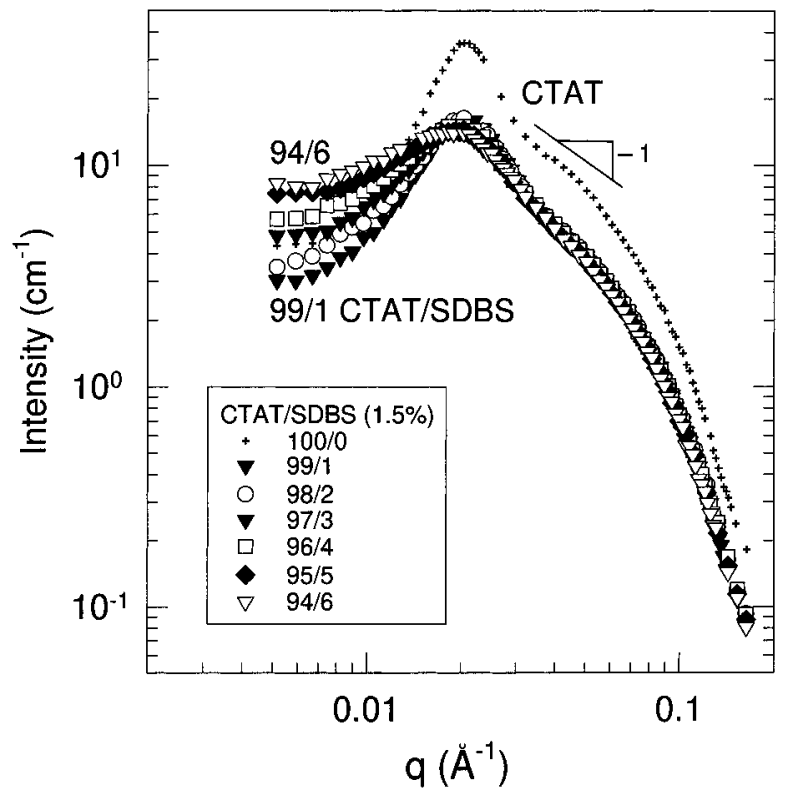

Figure 8. Effect of surfactant ratio on the static neutron scattering spectra of samples in $\mathrm{D}_{2} \mathrm{O}$. The spectra are plotted on an absolute scale.

distance between cylindrical micelles. The variation of $\xi$ with $\phi$ in the semidilute regime follows: ${ }^{40}$

$$
\xi=r_{\mathrm{cs}}\left(\frac{\pi}{\phi}\right)^{1 / 2}
$$

which describes the data (Figure 13). Next, the plateau modulus $G_{\infty}^{\prime}$ dictates the hydrodynamic correlation length $\xi_{\mathrm{H}}$ (i.e., the mesh size of the micellar network) through eq 6 . A plot of $\xi_{\mathrm{H}}$ against $\phi$ (Figure 13) follows the relation $\xi_{\mathrm{H}} \sim \phi^{-0.7}$ in line
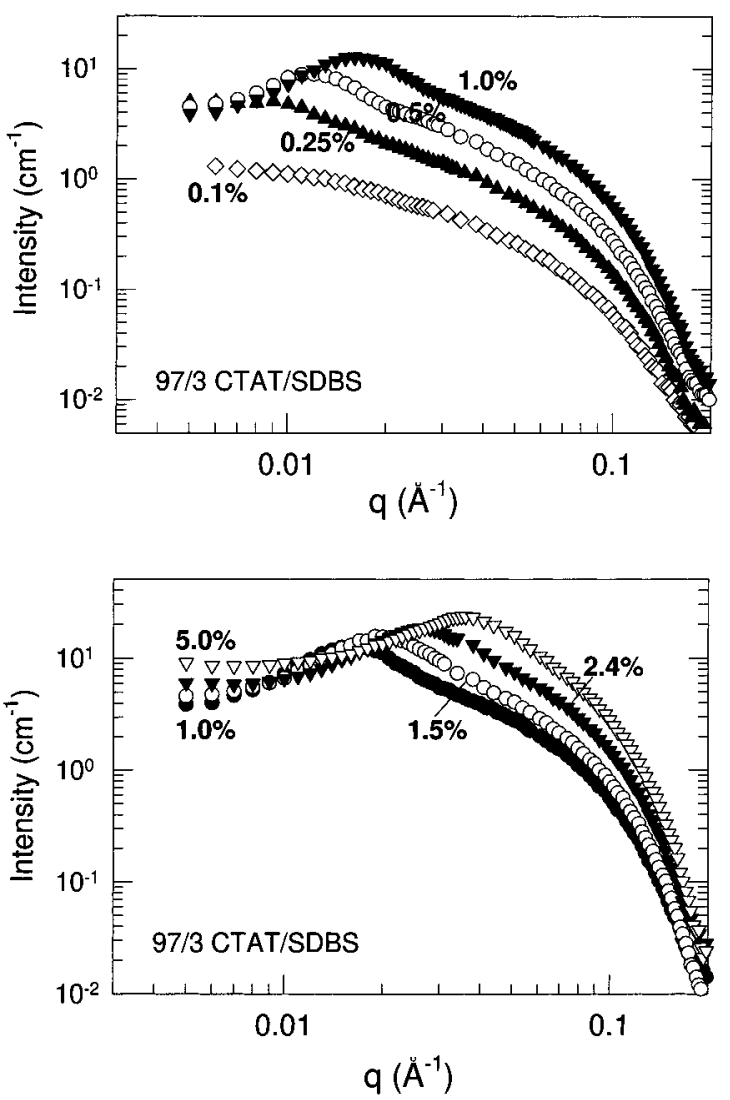

Figure 9. Effect of surfactant concentration on the static SANS spectra of samples in $\mathrm{D}_{2} \mathrm{O}$. The spectra are plotted on an absolute scale.

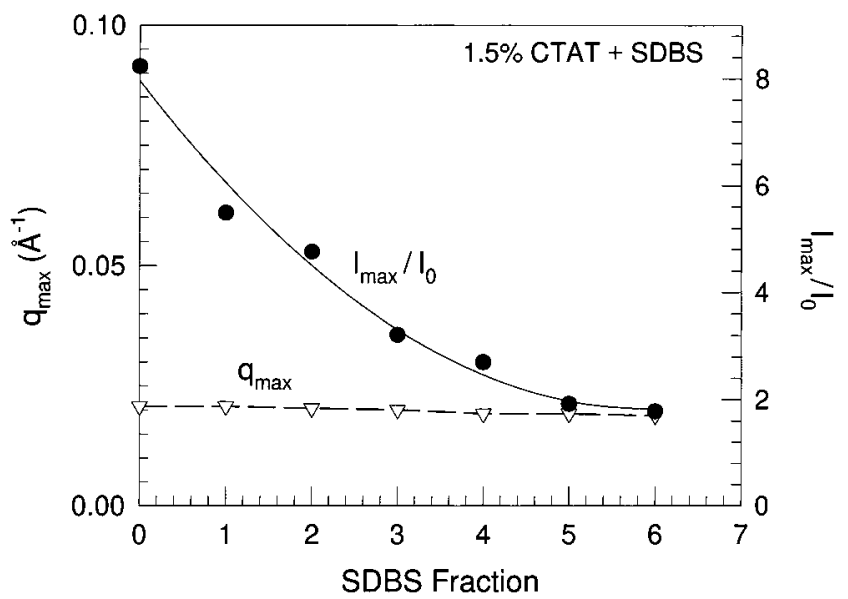

Figure 10. Analysis of SANS scattering peaks at various surfactant ratios. Data are shown for the peak position $q_{\max }$ and the peak height $\left(I_{\max } / I_{0}\right)$ as a function of the SDBS weight fraction in the added surfactant, i.e., $[\mathrm{SDBS}] /([\mathrm{CTAT}]+[\mathrm{SDBS}])$.

with $G_{\infty}^{\prime} \sim \phi^{2.1}$ (Figure 7). Thus, the mesh size $\xi_{\mathrm{H}}$ also decreases with concentration, but at a sharper rate than the mean separation $\xi$.

The persistence length $l_{\mathrm{p}}$, which characterizes the flexibility of the wormlike micelles, is the key remaining length scale. A description of $l_{\mathrm{p}}$ must account for the fact that wormlike micelles in salt-free solution behave as polyelectrolytes. Indeed, there are striking similarities between wormlike micelles and polyelectrolytes; for example, salt-free polyelectrolyte solutions also reveal a scattering peak in SANS, with their corresponding correlation length $\xi \sim \phi^{-1 / 2}$. $^{45}$ More significantly, the relaxation time $t_{\mathrm{R}}$ of polyelectrolyte solutions also shows an initial increase and then an extended decrease $\left(t_{\mathrm{R}} \sim \phi^{-1 / 2}\right)$ as a function of 


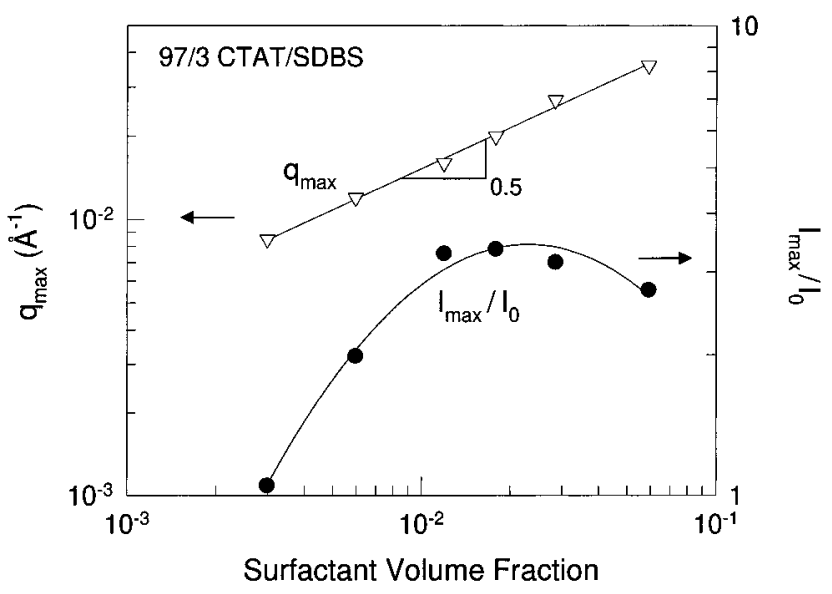

Figure 11. Analysis of SANS scattering peaks at various surfactant concentrations. Data are shown for the peak position $q_{\max }$ and the peak height $\left(I_{\max } / I_{0}\right)$ as a function of the total surfactant (CTAT + SDBS) volume fraction.

concentration. ${ }^{45,46}$ This effect is linked to the transition from rods to more compact coils as charge is screened at higher polymer concentrations.

The total persistence length for a polyelectrolyte chain is the sum of an intrinsic and an electrostatic contribution: $l_{\mathrm{p}}=l_{\mathrm{p}}{ }^{0}+$ $l_{\mathrm{p}}{ }^{\mathrm{e}} \cdot{ }^{4,47}$ The intrinsic persistence length $l_{\mathrm{p}}{ }^{0}$ can be relatively high ( $\sim 90 \AA$ ) for micelles because of their large cross-sections. ${ }^{4}$ The electrostatic persistence length $l_{\mathrm{p}}{ }^{\mathrm{e}}$ varies with the Debye length $\kappa^{-1}$ of the system, with the predictions being either $l_{\mathrm{p}}^{\mathrm{e}} \sim\left(\kappa^{-1}\right)^{2}$ (OSF theory ${ }^{47,48}$ ) or $l_{\mathrm{p}}^{\mathrm{e}} \sim\left(\kappa^{-1}\right)$ (Rubinstein et al. $\left.{ }^{49}\right)$. The Debye length for micellar solutions as a function of the surfactant concentration $c_{\mathrm{D}}$ and the salt concentration $c_{\mathrm{S}}$ is given by the following equation, with $l_{\mathrm{B}}$ being the Bjerrum length:4,5,38

$$
\kappa^{-1}=\left[4 \pi l_{\mathrm{B}} c_{\mathrm{D}}\left(\alpha+2 c_{\mathrm{s}} / c_{\mathrm{D}}\right)\right]^{-1 / 2}
$$

The micelle ionization degree $\alpha$ is predicted to increase monotonically with concentration for cylindrical micelles. ${ }^{5}$ Our estimates of $\alpha$ from SANS modeling indicate an initial increase and then a leveling off around $0.05 .{ }^{10}$ If $\alpha$ is constant and the salt concentration is low, $\kappa^{-1} \sim c_{\mathrm{D}}^{-1 / 2}$. On the basis of these equations, flexibility effects can be evaluated in our system. Increasing the SDBS fraction at constant $c_{\mathrm{D}}$ increases the

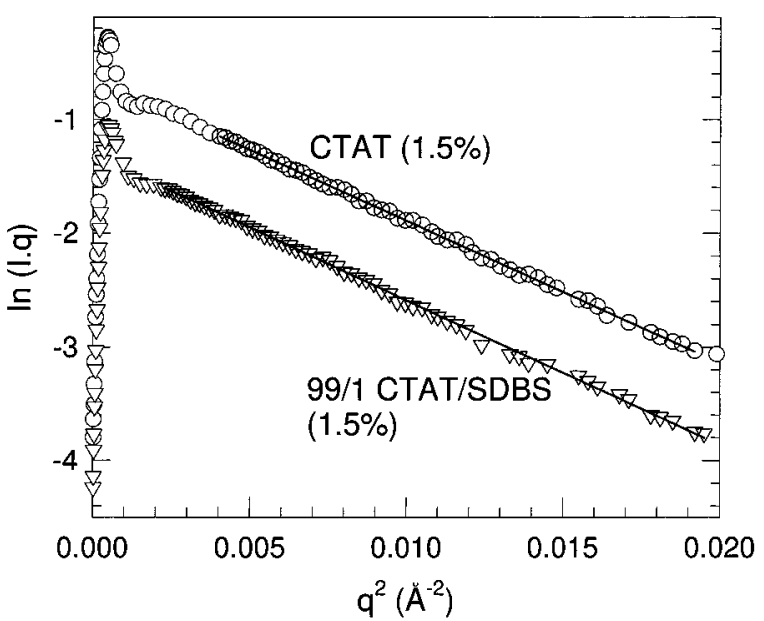

Figure 12. Cross-sectional Guinier plots for $1.5 \%$ CTAT and $1.5 \%$ CTAT/SDBS $99 / 1$ in $\mathrm{D}_{2} \mathrm{O}$. From the slopes of the fitted lines, the crosssectional radius $r_{\mathrm{cs}}$ of the micelles is found to be $22.5 \pm 0.5 \AA$, invariant of micellar composition.

concentration of free counterions, thus increasing the $c_{\mathrm{S}} / c_{\mathrm{D}}$ term and lowering $\kappa^{-1}$. An increase in total surfactant content at a constant SDBS/CTAT ratio fixes the $c_{\mathrm{S}} / c_{\mathrm{D}}$ term, but $\kappa^{-1}$ is lowered by the higher $c_{\mathrm{D}}$ (and by any increases in $\alpha$ ). In either case the decrease in $\kappa^{-1}$ results in a lower $l_{\mathrm{p}}^{\mathrm{e}}$ and hence a lower total $l_{\mathrm{p}}$. This implies that the micelles become more flexible at higher concentrations and at higher SDBS ratios.

In principle, $l_{\mathrm{p}}$ can be extracted from SANS or light scattering data (e.g., from inflection points in different representations of the scattering data ${ }^{4,42}$ ), but this is not possible here due to the influence of unscreened electrostatic interactions on the structure factor. An interesting possibility is to associate the SANS correlation length $\xi$ with the persistence length, as assumed in the Rubinstein theory ${ }^{49}$ for salt-free polyelectrolytes (since $l_{\mathrm{p}}$ $\approx l_{\mathrm{p}}^{\mathrm{e}} \sim \kappa^{-1} \sim c^{-1 / 2}$ which is the same scaling observed for $\xi$ ). However, the intrinsic persistence length $l_{\mathrm{p}}{ }^{0}$ may have a relatively large influence for micelles and, therefore, the equivalence between $\xi$ and the total $l_{\mathrm{p}}$ cannot be assumed. The lack of a constant $l_{\mathrm{p}}$ complicates the calculation of the micellar contour length $\bar{L}$. Moreover, the Cates model necessitates Maxwellian behavior of the micellar solutions, which is true only over limited ranges of concentration and composition

TABLE 1: Classification of Nonmonotonic Rheological Behavior Exhibited by Wormlike Micellar Systems

\begin{tabular}{|c|c|c|c|c|}
\hline class & observed phenomena & system & effect & examples \\
\hline \multirow[t]{5}{*}{ A } & $\mathbf{S A N S}^{a}$ & cationic + salt & $f($ salt $)$ & refs $2-4,21-24$ \\
\hline & $q_{\max }$ constant & cationic + anionic & $\begin{array}{l}f(\text { ratio }) \text { at fixed } \\
\text { total surfactant }\end{array}$ & this study \\
\hline & RHEOLOGY: & nonionic + anionic & " & ref 30 \\
\hline & & zwitterionic + anionic & " & ref 31 \\
\hline & $\begin{array}{l}G_{\min }^{\prime \prime} / G_{\infty}^{\prime} \text { shows minimum } \\
t_{\mathrm{R}}, \eta_{0} \text { show maximum }\end{array}$ & zwitterionic + cationic & " & ref 31 \\
\hline \multirow[t]{4}{*}{ B } & SANS $^{a}$ : & cationic & $f($ surfactant $)$ & refs $5-8,50$ \\
\hline & $q_{\max } \sim \phi^{0.5}$ & cationic + salt & $\begin{array}{l}f(\text { surfactant }) \text { at fixed salt } \\
\text { or at fixed ratio of } \\
\text { salt/surfactant }\end{array}$ & refs 3,24 \\
\hline & RHEOLOGY: & cationic + anionic & $\begin{array}{c}f(\text { surfactant }) \text { at fixed } \\
\text { surfactant ratio }\end{array}$ & this study \\
\hline & $\begin{array}{l}G_{\infty}^{\prime} \sim \phi^{\mathrm{a}}(a=1.7-2.3) \\
G_{\mathrm{min}}^{\prime \prime} / G_{\infty}^{\prime} \text { shows minimum } \\
t_{\mathrm{R}}, \eta_{0} \text { show maximum }\end{array}$ & nonionic + anionic & " & ref 30 \\
\hline
\end{tabular}

\footnotetext{
${ }^{a} q_{\max }$ corresponds to the peak in the SANS spectra that is observed only in absence of salt.
} 


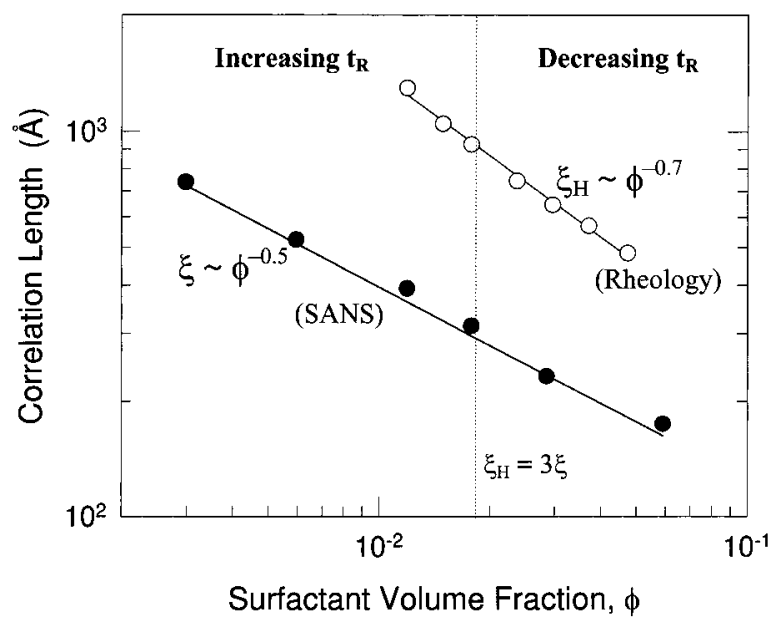

Figure 13. Comparison of correlation lengths obtained from SANS and rheology for $97 / 3$ CTAT/SDBS micelles. The filled points represent the values of the SANS correlation length $\xi$ obtained from the scattering peak positions and the straight line through the points is a plot of eq 14. The unfilled points represent the hydrodynamic correlation lengths $\xi_{\mathrm{H}}$ obtained from the plateau modulus $G_{\infty}^{\prime}$ using eq 6 and the straight line is a best fit through the points. At the point of maximum relaxation time $t_{\mathrm{R}}, \xi_{\mathrm{H}}$ is observed to be $\approx 3 \xi$.

(Figures 4,7 ). Therefore, only qualitative trends in $\bar{L}$ can be identified, assuming eqs 4 and 5 to be valid.

Surfactant Concentration. Consider first the effect of surfactant concentration $\phi$ on $\bar{L}$. If $l_{\mathrm{p}}$ is constant, the entanglement length $l_{\mathrm{e}} \sim \xi_{\mathrm{H}}^{5 / 3} \sim \phi^{-1.16}$. If instead $l_{\mathrm{p}}$ is a function of concentration, scaling either as $\phi^{-0.5}$ (Rubinstein) or $\phi^{-1}$ (OSF), then by eq $5, l_{\mathrm{e}}$ would scale as $\phi^{-0.83}$ or as $\phi^{-0.5}$, respectively. In all cases, $l_{\mathrm{e}}$ is a weakly decreasing function, which means that $\bar{L}(\phi)$ is basically related to $\left(G_{\min }^{\prime \prime} / G_{\infty}^{\prime}\right)^{-1}$, i.e., the $\bar{L}(\phi)$ plot is an inversion of the $G_{\min }^{\prime \prime} / G_{\infty}^{\prime}$ vs $\phi$ plot (Figure 7b). The minimum in $G_{\min }^{\prime \prime} / G_{\infty}^{\prime}$ as a function of $\phi$ therefore implies a maximum in $\bar{L}$ at the corresponding $\phi$, suggesting that the micelles grow to an optimum length and thereafter become shorter.

The maximum in $\bar{L}$ follows from the application of the Cates model to the rheology data; however, the validity of the model may be questioned. Consider, in this regard, the micellar breaking time $\tau_{\mathrm{b}}$ which $\sim \bar{L}^{-1}$ in the Cates model, meaning that longer micelles break more quickly. A rough indication of $\tau_{\mathrm{b}}$ is given by the inverse of the frequency $\omega_{\mathrm{b}}$ at which $G^{\prime \prime}$ shows a minimum. Although the shallow nature of the $G^{\prime \prime}$ curve around its minimum point precludes accurate estimation of $\omega_{\mathrm{b}}$, Figure $5 \mathrm{~b}$ clearly reveals a shift in $\omega_{\mathrm{b}}$ to higher frequencies for concentrated samples, implying that $\tau_{\mathrm{b}}$ decreases with $\phi$ in this regime. A decrease in $\bar{L}$ with $\phi$ in the same regime would mean that the micelles are becoming shorter and breaking more easily-in violation of the earlier assumptions.

Several studies on salt-free cationic surfactants have interpreted the rheology maxima in terms of a maximum in the length of linear micelles, despite similar anomalies concerning the trends in $\tau_{\mathrm{b}}{ }^{5,7,8}$ Thus, these studies report a decreasing $\tau_{\mathrm{b}}(\phi)$ in the high concentration range where the solutions are Maxwell fluids and the Cates model predicts a decreasing $\bar{L}(\phi)$. At lower concentrations, they report a steep viscosity rise with a power law exponent much higher than the $3 / 2$ predicted for screened micelles (eq 8); indeed, we correspondingly find an exponent ca. 9 (Figure 7b). The higher exponent is related to the rapid increase in the length of charged micelles at low concentrations (see eq 16). ${ }^{5,38}$ Moreover, at low $\phi$, the system may be in the slow breaking regime $\left(\tau_{\mathrm{b}}>\tau_{\text {rep }}\right)$ due to the small micellar size, and in this regime theory ${ }^{5}$ predicts $\eta_{0} \sim \phi^{3} \bar{L}^{6}$.
Alternately, the maximum in $\eta_{0}(\phi)$ may be attributed to micellar branching; thus the micelles are expected to be linear in the regime of increasing $\eta_{0}$ and branched in the regime of decreasing $\eta_{0}{ }^{5,8}$ For branched or cross-linked micelles, the $\bar{L}$ derived from rheology data is interpreted as a characteristic arc length $\bar{L}_{\mathrm{c}}$ of the cross-linked micellar network. ${ }^{21}$ However, the breaking time $\tau_{\mathrm{b}}$ should still vary inversely with $\bar{L}_{\mathrm{c}}$; thus, a simultaneous decrease in $\tau_{\mathrm{b}}$ and $\bar{L}_{\mathrm{c}}$ at high $\phi$ is inconsistent with branching as well. ${ }^{5}$ Moreover, recent studies on a trimeric surfactant by In et al., ${ }^{50}$ which revealed branched micelles under cryo-TEM, found an anomalous scaling relationship for the plateau modulus $G_{\infty}^{\prime}(\phi)$ (a much steeper variation than the $\sim$ $\phi^{2.25}$ observed typically). The authors also studied a dimeric surfactant which manifested only linear micelles under cryoTEM. While both surfactants gave rise to viscosity maxima as a function of $\phi$, only the trimeric system showed the anomalous scaling of $G_{\infty}^{\prime}$. This suggests that the nonmonotonic behavior of $\eta_{0}(\phi)$ cannot, as a general rule, be attributed to branching.

A maximum in linear contour length $\bar{L}$ is therefore the more likely explanation, and changes in $\bar{L}$ must be driven by changes in the parameters controlling self-assembly, in particular, the scission energy $E_{\text {scis. }}$. For neutral or screened micelles, $E_{\text {scis }}$ is equal to the end-cap energy $E_{\mathrm{c}}$, and the micellar length $\bar{L}(\phi)$ is given by eq 1 . For charged micelles, in addition to the end-cap energy $E_{\mathrm{c}}$ which favors micellar growth, there is an electrostatic contribution $E_{\mathrm{e}}$ to the effective scission energy which favors the breaking of the micelles. ${ }^{38,51}$ The modified expression for $\bar{L}(\phi)$ in the semidilute regime is ${ }^{51}$

$$
\bar{L} \sim \phi^{1 / 2} \exp \left[\frac{1}{2 k_{\mathrm{B}} T}\left(E_{\mathrm{c}}-E_{\mathrm{e}}\right)\right]
$$

The electrostatic contribution $E_{\mathrm{e}}$, which arises due to the repulsive energy of the surface charges, is given by

$$
E_{\mathrm{e}} \cong \frac{k_{\mathrm{B}} T l_{\mathrm{B}} r_{\mathrm{cs}} v^{2}}{\phi^{1 / 2}}
$$

Here $v$ is an effective charge per unit length. Kern et al. ${ }^{5}$ speculated that $v$ may increase with $\phi$ because of the screening introduced by other micelles, thus tending to increase $E_{\mathrm{e}}$. On the other hand, an increase in the effective micellar charge may reduce the end-cap energy $E_{\mathrm{c}}$ itself (this may be linked to the number of condensed counterions per surfactant). ${ }^{5}$ Both these effects will tend to inhibit micellar growth and reduce $\bar{L}$.

If indeed the micellar contour length $\bar{L}$ (and equivalently, rheological properties such as $t_{\mathrm{R}}$ and $\eta_{0}$ ) reach an optimum, the challenge is to predict the concentration $\phi_{\max }$ at which this occurs. The governing factor is speculated to be the ratio of $\phi_{\max }$ to the overlap concentration $\phi^{*} .{ }^{8,50,51}$ Experimental values of $\phi_{\max } / \phi^{*}$ range from 2.3 to 10 with inconsistencies in the definition of $\phi_{\max }$ and $\phi^{*}$ contributing to the breadth of this range. In our system, defining $\phi_{\max }$ as the concentration where $t_{\mathrm{R}}$ reaches a maximum, we find $\phi_{\max } / \phi^{*} \approx 6$ which is consistent with the value found by In et al. ${ }^{50}$ for their salt-free surfactant solutions. The ratio $\phi_{\max } / \phi^{*}$ appears to capture some of the correct qualitative trends including the effect of temperature on the position of the viscosity maxima. Thus, increasing the temperature causes a rise in $\phi^{*}\left(\right.$ theory $^{5}$ predicts $\phi^{*} \sim T^{10 / 3}$ ) and there is correspondingly an increase in $\phi_{\max }$ also, with a constant value of $\phi_{\max } / \phi^{*}$ being nearly maintained..$^{5,8}$ The critical value of $\phi_{\max } / \phi^{*}$ may represent the point when the end-cap energy $E_{\mathrm{c}}$ overcomes the electrostatic energy $E_{\mathrm{e}}{ }^{8}$

An alternate possibility is that $\phi_{\max }$ is related to characteristic length scales, in particular to a ratio of $\xi_{\mathrm{H}}$ to $\xi$. The hydrody- 
namic correlation length $\xi_{\mathrm{H}}$ from rheology reflects the mesh size of the entangled network. The SANS correlation length $\xi$ corresponds to a mean distance between rigid rods, and it can be viewed as an electrostatic correlation length, as assumed for polyelectrolytes. ${ }^{49}$ The fact that $\xi_{\mathrm{H}}$ is typically larger than $\xi$ implies that not every interchain contact point represents a topological constraint. The ratio $\xi_{\mathrm{H}} / \xi$ continuously decreases with $\phi$ (Figure 13) and our empirical observation is that $\xi_{\mathrm{H}} / \xi$ $\approx 3$ at $\phi_{\max }$. Inspection of the data reported for other salt-free micellar systems ${ }^{7,8}$ shows this relationship to be generally valid. An interpretation is that at this critical ratio between the hydrodynamic and electrostatic length scales, the micelles may be so close that not only do their counterion clouds overlap, but also the micelles may themselves be acting as polyions and contributing to the electrostatic screening. ${ }^{49}$

Polyion screening may, in turn, explain why the SANS peak height also shows an overturn in the vicinity of $\phi_{\max }$ (Figure 9). The SANS scattering peak arises due to intermicellar repulsions. At low surfactant concentrations, the size and density of charged entities is low, which is why the peak is absent initially. The average distance between micelles decreases as the micelles grow and at the onset of the semidilute regime $\left(c^{*}\right)$, the micellar size is equal to the mean distance between rods, i.e., $\bar{L} \approx \xi$, at which point a scattering peak first appears. Further increase in surfactant content leads to an overlap in micellar screening lengths, thus enhancing the peak height. The decrease in peak height at higher concentrations would result from polyion screening. Simulations of scattering functions for rodlike polyelectrolytes ${ }^{41}$ also appear to show a decrease in peak height at high $c / c^{*}$. A maximum in SANS peak height $\left(I_{\max } / I_{0}\right)$ with concentration was observed earlier by Hoffmann et al. for solutions of a catanionic surfactant, ${ }^{43}$ although the effect was not correlated with the solution rheology.

Our results demonstrate clear consistency between the static properties of the system, as measured with SANS, and the dynamic properties, as estimated from rheology. Unresolved issues include the different scaling exponents exhibited by micelles for $\xi$ and $\xi_{\mathrm{H}}$ : the former $\sim \phi^{-1 / 2}$ as for polyelectrolytes, while the latter $\sim \phi^{-3 / 4}$ as for neutral polymers. In et al. ${ }^{50}$ recently suggested that the system is fully entangled only beyond the rheology maxima - at lower concentrations, the mesh size may depend on micellar length implying that the system is not strictly in the semidilute regime. The precise nature of the micellepolymer analogy thus remains an open theoretical question.

Surfactant Composition. Moving to the effect of surfactant composition, the explanation for the viscosity maximum as a function of the SDBS fraction (Figure 4a) again centers around one or more of the following microstructural effects: branching, length changes, or flexibility changes. The possibility of micellar branching at high salt content was discussed in the Introduction, and considering the analogy between the composition results and salt effects (Table 1), the same hypothesis may be valid here also. For branching to occur, cross-links must become energetically favorable over hemispherical end-caps, and this may indeed be the case when oppositely charged surfactants are combined. More quantitatively, consider the surfactant packing parameter $p=v / l a$ where $v$ is the hydrophobic volume, $l$ is the hydrophobic chain length, and $a$ is the headgroup area. A value of $p$ between $1 / 3$ and $1 / 2$ implies formation of cylindrical micelles, while $p$ between $1 / 2$ and 1 implies flexible bilayers. ${ }^{52}$ Strong interaction between anionic and cationic surfactant molecules causes an increase in $v$ and a decrease in $a{ }^{52-54}$ Thus, increasing the SDBS fraction increases $p$, which tends to promote cross-links over the highly curved end-caps. ${ }^{28}$
Moreover, the phase diagram (Figure 1) confirms that further increases in SDBS content beyond that investigated here eventually causes a transition to bilayer phases - consistent with further increases in the packing parameter $p$ to values approaching 1.9 Before forming bilayer phases, the branched micelles first undergo phase separation, which is also consistent with the theoretical predictions of Drye and Cates. ${ }^{26}$ Evidence from cryo-TEM also suggests that branched micelles can form in a mixed cationic/anionic system. ${ }^{28}$

An alternate explanation for these results focuses on the analogy with salt effects in polyelectrolytes. Experiments on polyelectrolyte solutions reveal that $\eta_{0}$ is initially independent of salt, but decreases at high salt concentrations. ${ }^{46}$ This decrease is associated with the increased flexibility of chains on adding salt. A similar scenario may be envisioned for wormlike micelles. Note that when SDBS is incorporated into CTAT micelles, there is the concomitant release of "salt" formed from the oppositely charged counterions into the bulk solution. Assuming that all of the SDBS is in the micelles, ${ }^{10}$ the concentration of salt generated is proportional to the SDBS concentration. Increasing the SDBS fraction thus introduces more salt, decreasing $\kappa^{-1}$ (eq 15) and thereby lowering $l_{\mathrm{p}}$ e. The penetrating SDBS molecules may also lower the bending modulus of the mixed micelle $\mathrm{e}^{55}$ and thereby its intrinsic persistence length $l_{\mathrm{p}}{ }^{0}$. Both these factors imply a reduction in $l_{\mathrm{p}}$, i.e., more flexible micelles, at high SDBS content.

These changes in micelle properties will be reflected in the rheology. At low SDBS content, flexibility effects may be masked by strong electrostatic interactions between pairs of oppositely charged surfactant headgroups. The lowering of repulsions between headgroups favors growth into longer micelles, which contribute to a higher viscosity. At high SDBS content, the increase in micellar flexibility may become the dominant effect, thus reducing the viscosity, as is the case for polyelectrolytes. Thus a scenario based on an initial increase in micellar length with added SDBS to an optimum value, followed by an enhancement in micellar flexibility at higher SDBS content can explain the rheological data.

There are thus two conflicting hypotheses that could account for the overturn in viscosity with varying surfactant composition. The branching hypothesis is certainly more prevalent in the literature, but it is by no means definitive. For example, cryoTEM studies by Clausen et al. ${ }^{22}$ found no evidence for branched micelles even at high salt. The polyelectrolyte-micelle analogy is certainly interesting, and worthy of further theoretical investigation such as by applying the theory of Rubinstein et al. ${ }^{49}$ to micelles. A comprehensive theory should also be able to explain other experimental intricacies (e.g., a second viscosity maximum observed for certain surfactant/salt combinations), for which no explanation presently exists.

\section{Conclusions}

Addition of small amounts of anionic SDBS to cationic CTAT promotes the growth of wormlike micelles and thereby enhances the viscoelastic character of the system. This synergy is brief, however, since at higher SDBS fractions, the rheological properties are diminished. There are two possible explanations for this phenomenon. Considering the polyelectrolyte nature of the wormlike micelles, the rise in ionic strength at high SDBS content may lead to more flexible chains which would then be able to relax faster. Alternately, the strong mutual interaction between cationic and anionic counterparts may facilitate a transition from linear micelles to a branched micellar network of reduced viscosity. 
Increasing the concentration of surfactant at a fixed composition of the mixed micelles again reveals an optimal rheological behavior at an intermediate concentration. The solutions also show a scattering peak in SANS and the peak height also exhibits a similar nonmonotonic trend with concentration. The most probable microstructural explanation for these phenomena is that the micellar contour length goes through a maximum. It is likely that the optimal contour length corresponds to a delicate balance in the length scales representing hydrodynamic and electrostatic screening.

Acknowledgments. The authors acknowledge NIST for providing the facilities for SANS experiments, and the assistance of J. A. Silas and D. J. Iampietro in performing these experiments. Helpful discussions with Prof. R. de Vries are also acknowledged. Financial support for this work came from the NSF-CTS 9814399

\section{References and Notes}

(1) Cates, M. E.; Candau, S. J. J. Phys. Condens. Matter 1990, 2, 6869.

(2) Rehage, H.; Hoffmann, H. Mol. Phys. 1991, 74, 933.

(3) Hoffmann, H. In Structure and Flow in Surfactant Solutions; ACS Symp. Ser. 578; Herb, C. A., Prudhomme, R., Eds.; ACS: Washington, DC, 1994; pp 2-31.

(4) Magid, L. J. J. Phys. Chem. 1998, 102, 4064. 1714 .

(5) Kern, F.; Lequeux, F.; Zana, R.; Candau, S. J. Langmuir 1994, 10 ,

(6) Soltero, J. F. A.; Puig, J. E. Langmuir 1996, 12, 2654.

(7) Carver, M.; Smith, T. L.; Gee, J. C.; Delichere, A.; Caponetti, E.; Magid, L. J. Langmuir 1996, 12, 691.

(8) Oda, R.; Narayanan, J.; Hassan, P. A.; Manohar, C.; Salkar, R. A.;

Kern, F.; Candau, S. J. Langmuir 1998, 14, 4364.

(9) Kaler, E. W.; Herrington, K. L.; Murthy, A. K.; Zasadzinski, J. A. N. J. Phys. Chem. 1992, 96, 6698 .

(10) Koehler, R. D. Ph.D. Thesis, University of Delaware, 1995

(11) Koehler, R. D.; Kaler, E. W. In Structure and Flow in Surfactant Solutions; ACS Symp. Ser. 578; Herb, C. A., Prudhomme, R., Eds.; ACS: Washington, DC, 1994; pp 2-31.

(12) Cates, M. E. Macromolecules 1987, 20, 2289

(13) Granek, R.; Cates, M. E. J.Chem. Phys. 1992, 96, 4758

(14) Cates, M. E. J. Phys. Condens. Matter 1996, 8, 9167.

(15) Larson, R. G. The Structure and Rheology of Complex Fluids; Oxford University Press: Oxford, 1999.

(16) Doi, M.; Edwards, S. F. The Theory of Polymer Dynamics; Clarendon Press: Oxford, 1986.

(17) Kern, F.; Lemarechal, P.; Candau, S. J.; Cates, M. E. Langmuir 1992, 8, 437.

(18) Lequeux, F.; Candau, S. J. In Structure and Flow in Surfactant Solutions; ACS Symp. Ser. 578; Herb, C. A., Prudhomme, R., Eds.; ACS: Washington, DC, 1994; pp 51-62.

(19) Lequeux, F. Curr. Opin. Colloid Interface Sci. 1996, 1, 341.

(20) Candau, S. J.; Lequeux, F. Curr. Opin. Colloid Interface Sci. 1997, 2,420 .

(21) Khatory, A.; Lequeux, F.; Kern, F.; Candau, S. J. Langmuir 1993, 9, 1456.

(22) Clausen, T. M.; Vinson, P. K.; Minter, J. R.; Davis, H. T.; Talmon, Y.; Miller, W. G. J. Phys. Chem. 1992, 96, 474
(23) Aswal, V. K.; Goyal, P. S.; Thiyagarajan, P. J. Phys. Chem. B 1998 , 102,2469

(24) Ait Ali, A.; Makhloufi, R. Colloid Polym. Sci. 1999, 277, 270

(25) Lequeux, F. Europhys. Lett. 1992, 19, 675.

(26) Drye, T. J.; Cates, M. E. J. Chem. Phys. 1992, 96, 1367.

(27) Danino, D.; Talmon, Y.; Levy, H.; Beinert, G.; Zana, R. Science 1995, 269, 1420 .

(28) Lin, Z. Langmuir 1996, 12, 1729.

(29) Swanson-Vethamuthu, M.; Almgren, M.; Karlsson, G.; Bahadur, P. Langmuir 1996, 12, 2173.

(30) Herb, C. A.; Chen, L. B.; Sun, W. M. In Structure and Flow in Surfactant Solutions; ACS Symp. Ser. 578; Herb, C. A., Prudhomme, R., Eds.; ACS: Washington, DC, 1994; pp 153-166.

(31) Hoffmann, H.; Rauscher, A.; Gradzielski, M.; Schulz, S. F. Langmuir 1992, 8, 2140.

(32) Zhao, G. X.; Xiao, J. X. Colloid Polym. Sci. 1995, 273, 1088.

(33) Rosen, M. J. In Mixed Surfactant Systems; ACS Symp. Ser. 501; Holland, P. M., Rubingh, D. N., Eds.; ACS: Washington, DC, 1992; pp $316-326$

(34) Barker, C. A.; Saul, D.; Tiddy, G. J. T.; Wheeler, B. A.; Willis, E. J. Chem. Soc., Faraday Trans. 1 1974, 70, 154. 439

(35) Kato, T.; Iwai, M.; Seimiya, T. J. Colloid Interface Sci. 1989, 130,

(36) Berret, J.-F.; Gamez-Corrales, R.; Oberdisse, J.; Walker, L. M.; Lindner, P. Europhys. Lett. 1998, 41, 677.

(37) Gamez-Corrales, R.; Berret, J.-F.; Walker, L. M.; Oberdisse, J. Langmuir 1999, 15, 6755.

(38) Mackintosh, F. C.; Safran, S. A.; Pincus, P. A. Europhys. Lett. 1990, 12,697

(39) Schurtenburger, P.; Scartazzini, R.; Magid, L. J.; Leser, M. E.; Luisi, P. L. J. Phys. Chem. 1990, 94, 3695

(40) Chen, S. H.; Sheu, E. Y.; Kalus, J.; Hoffmann, H. J. Appl. Crystallogr. 1988, 21, 751 .

(41) Weyerich, B.; D’Aguanno, B.; Canessa, E.; Klein, R. Faraday Discuss. Chem. Soc. 1990, 90, 245.

(42) Butler, P. D.; Magid, L. J.; Hayter, J. B. In Structure and Flow in Surfactant Solutions; ACS Symp. Ser. 578; Herb, C. A., Prudhomme, R., Eds.; ACS: Washington, DC, 1994; pp 250-257.

(43) Hoffmann, H.; Kalus, J.; Schwandner, B. Ber. Bunsen-Ges. Phys. Chem. 1987, 91, 99.

(44) Tanford, C. The Hydrophobic Effect: Formation of Micelles and Biological Membranes, 2nd ed.; John Wiley \& Sons: New York, 1980.

(45) Krause, W. E.; Tan, J. S.; Colby, R. H. J. Polym. Sci., Part B 1999, $37,3429$.

(46) Boris, D. C.; Colby, R. H. Macromolecules 1998, 31, 5746.

(47) Odijk, T. J. Polym. Sci., Part B 1977, 15, 477.

(48) Skolnick, J.; Fixman, M. Macromolecules 1977, 10, 944

(49) Dobrynin, A. V.; Colby, R. H.; Rubinstein, M. Macromolecules 1995, 28, 1859 .

(50) In, M.; Warr, G. G.; Zana, R. Phys. Rev. Lett. 1999, 83, 2278.

(51) Narayanan, J.; Manohar, C.; Kern, F.; Lequeux, F.; Candau, S. J. Langmuir 1997, 13, 5235.

(52) Israelachvili, J. Intermolecular and Surface Forces; Academic Press: San Diego, 1991.

(53) Herrington, K. L.; Kaler, E. W.; Miller, D. D.; Zasadzinski, J. A.; Chiruvolu, S. J. Phys. Chem. 1993, 97, 13792

(54) Brasher, L. L.; Herrington, K. L.; Kaler, E. W. Langmuir 1995, $11,4267$.

(55) Magid, L. J.; Han, Z.; Li, Z.; Butler, P. D. J. Phys. Chem. B 2000, $104,6717$. 